\title{
Simulations of the dust acoustic instability in a collisional plasma with warm dust
}

\author{
K. Quest ${ }^{1}$, M. Rosenberg ${ }^{1} \dagger$, B. Kercher ${ }^{1}$ and M. Dutreix ${ }^{1}$ \\ ${ }^{1}$ Department of Electrical and Computer Engineering, University of California San Diego, \\ La Jolla, CA 92093, USA
}

(Received 22 July 2016; revised 18 October 2016; accepted 18 October 2016)

Dust acoustic (or dust density) waves have been observed in many laboratory dusty plasmas. These low-frequency waves involve the dynamics of highly charged and massive dust grains, and can be excited by the flow of ions relative to dust. In this paper, we consider the nonlinear development of the dust acoustic instability, excited by thermal ion flow, in a collisional plasma containing dust with high kinetic temperature (warm dust). It is shown that under certain conditions there may be a long-wavelength secondary instability in the nonlinear stage as dust gets heated by the waves. The characteristics of the nonlinear development are considered as a function of the relative charge density of the dust. Application to possible experimental parameters is discussed.

Key words: dusty plasmas, plasma waves

\section{Introduction}

Dusty plasmas are ionized gases containing small (nanometre- to micron-sized) charged solid particulates or dust grains. A fundamental low-frequency collective mode in such plasmas is the dust acoustic (DA) wave (also referred to as a dust density wave). The charged dust provides the inertia and the background electrons and ions provide the pressure to sustain this wave (Rao, Shukla \& Yu 1990). The DA wave generally has frequency less than the dust plasma frequency $\omega_{p d}$ and phase speed on the order of the dust acoustic speed $c_{d} \sim \lambda_{D i} \omega_{p d}\left(\lambda_{D i}\right.$ is the ion Debye length), which is much smaller than the ion thermal speed. In typical laboratory dusty plasma experiments, DA waves have frequencies in the range of $10 \mathrm{~Hz}$, wavelengths in the range of millimetres and phase speeds in the range of $\mathrm{cm} \mathrm{s}^{-1}$. Dust acoustic waves have been observed in many dusty plasma experiments both in the terrestrial laboratory and under microgravity conditions (Merlino 2014). It is thought that these waves are excited by ion flow via an instability referred to as a dust acoustic instability (e.g. Rosenberg 1993) or, more generally, an ion-dust streaming instability, which also includes the case when the ion flow is superthermal (e.g. Rosenberg 1996; Joyce, Lampe \& Ganguli 2002).

One parameter which influences both the dispersion and excitation of DA waves is the thermal energy of the dust, that is, the dust kinetic temperature $T_{d}$. A number

$†$ Email address for correspondence: rosenber@ece.ucsd.edu 
of dusty plasma experiments have been performed to measure the velocity space distribution function of dust in dc glow discharge dusty plasmas (e.g. Williams \& Thomas 2006, 2007). It was observed that the dust kinetic temperature was high and could be greater than that of any other plasma component. In addition, a comparison of the linear kinetic theory of the ion-dust streaming instability with experimental measurements of the dispersion relation of DA waves showed that it was necessary to take into account the effects of dust with high kinetic temperature, i.e. 'warm' dust (Rosenberg, Thomas \& Merlino 2008). By warm dust, we mean here that the dust acoustic speed $c_{d}$ is not much larger than the dust thermal speed. While the reason for the large dust kinetic temperature is not definitively known, theories for dust heating have been proposed, including dust heating by wave-particle interactions (e.g. Melzer, Homann \& Piel 1996; Schweigert et al. 1996; Joyce et al. 2002). In addition, numerical particle-in-cell (PIC) simulations have shown that dust can get heated by waves in the nonlinear development of the ion-dust streaming instability (Winske et al. 1995; Winske \& Rosenberg 1998; Winske 2004; Fu \& Scales 2012).

In this paper, we present one-dimensional (1D) PIC simulations of the nonlinear development of the dust acoustic instability, taking into account the effects of warm dust. We also include collisions of charged particles with neutrals, which is a dominant collisional process at the higher pressures typical of dusty plasma experiments, and an electric field that drives the streaming of ions relative to dust. While in this sense our simulation approach is similar to that of Winske (2004) for a periodic system, here we consider effects of warm dust in both the linear and nonlinear phases. In addition, we consider the parameter regime where the ion flow speed is thermal, which may be relevant to certain ground-based experiments with high pressure, but particularly to dusty plasma experiments in microgravity. In microgravity, smaller electric fields are needed to confine the dust, which implies that the ion flow speeds can generally be smaller than in ground-based experiments. We also investigate how the characteristics of the nonlinear development of the instability vary as the relative charge density of the dust varies.

The paper is organized as follows. In $\S 2$, a linear kinetic dispersion relation for the ion-dust streaming instability for a range of reduced parameters is given. Numerical simulations of the linear and nonlinear development of the ion-dust streaming instability are presented in $\$ 3$ for these parameters. Section 4 gives a summary and discussion.

\section{Linear theory}

The model system that we consider is a weakly ionized plasma composed of electrons, singly charged ions and negatively charged dust grains of uniform radius $R$. The condition of overall charge neutrality is

$$
n_{e}+Z_{d} n_{d}=n_{i}
$$

Here, the subscripts $e, i$ and $d$ refer to electrons, ions and dust, respectively, and $Z_{d}$ is the charge state of the dust. There is a background neutral gas of density $n_{n}$, and an external constant electric field $E_{0}$ which induces drifts in the charged-particle species. We assume that the drift velocity is roughly given by $\boldsymbol{U}_{j}=q_{j} \boldsymbol{E}_{0} / m_{j} v_{j}$, where $q_{j}$ and $m_{j}$ are the charge and mass of each charged-particle species, and $v_{j}$ is the collision frequency of each charged-particle species with the background neutral atoms. We will consider the frame where there is no dust drift. (In an experimental situation, this may 


$\begin{array}{lcc}P & 20 \mathrm{~Pa} & \\ n_{i} & 2 \times 10^{8} \mathrm{~cm}^{-3} & T_{e} / T_{i}=167 \\ m_{i} & 20 \mathrm{~m}_{p} & v_{i} / \omega_{p i}=0.5 \\ T_{e} & 5 \mathrm{eV} & v_{e} / \omega_{p i}=30 \\ T_{i} & 0.03 \mathrm{eV} & Z_{d}=1.5 \times 10^{3} \\ R & 0.65 \mu \mathrm{m} & m_{d} / m_{i}=5 \times 10^{10} \\ n_{d} & 1 \times 10^{5} \mathrm{~cm}^{-3} & n_{d} / n_{i}=5 \times 10^{-4} \\ m_{d} & 1 \times 10^{12} \mathrm{~m}_{p} & \omega_{p d} / \omega_{p i}=1.5 \times 10^{-4} \\ \lambda_{D i} & \sim 100 \mu \mathrm{m} & v_{d} / \omega_{p d}=0.2\end{array}$

TABLE 1. Nominal parameters.

be due to a balance of the electric force acting on the dust with the ion drag force which acts in the opposite direction.)

For this system, assuming that the particle velocity distributions can be described by drifting Maxwellians, the dispersion relation of the ion-dust streaming instability can be taken as (see e.g. Miyamoto 1989; Rosenberg 1996)

$$
1+\sum_{j} \frac{1}{k^{2} \lambda_{D j}^{2}} \frac{\left[1+\zeta_{j} Z\left(\zeta_{j}\right)\right]}{\left[1+\left(i v_{j} / \sqrt{2} k v_{j}\right) Z\left(\zeta_{j}\right)\right]}=0 .
$$

Here, $\lambda_{D j}=\left(T_{j} / 4 \pi n_{j} q_{j}^{2}\right)^{1 / 2}$ is the Debye length, $v_{j}=\left(T_{j} / m_{j}\right)^{1 / 2}$ is the thermal speed and $Z(\zeta)$ is the plasma dispersion function (Fried \& Conte 1961) with arguments

$$
\zeta_{e, i}=\frac{\omega-\boldsymbol{k} \cdot \boldsymbol{U}_{e, i}+\mathrm{i} v_{e, i}}{\sqrt{2} k v_{e, i}}, \quad \zeta_{d}=\frac{\omega+\mathrm{i} v_{d}}{\sqrt{2} k v_{d}} .
$$

We consider a 1D system, with the wavevector $\boldsymbol{k}$ in the direction of the ion drift, which we take to be in the $x$-direction. The $1 \mathrm{D}$ approximation is reasonable for the case that we consider in this paper where the ion drift speed is comparable to the ion thermal speed, since the fastest-growing waves would be expected to propagate along the ion streaming direction.

To solve (2.2), we consider a set of nominal dusty plasma parameters that may be within the range of possible dusty plasma experiments in PK-4 microgravity conditions (Fortov et al. 2005; Khrapak et al. 2005). These parameters with a neon plasma are listed in table 1 , along with corresponding approximate dimensionless parameters. The dust charge was estimated using the orbit-motion-limited approximation, but with a reduction of about $1 / 3$ due to ion collisional effects (see Khrapak et al. 2005). To obtain the electron and ion collision frequencies, we used $v_{e, i} \sim \sigma_{e, i} n_{n} v_{e, i}$ with $\sigma_{e} \sim$ $2.5 \times 10^{-16} \mathrm{~cm}^{2}$ and $\sigma_{i} \sim 1 \times 10^{-14} \mathrm{~cm}^{2}$. The collision rate of dust with neutrals is given by Khrapak, Ivlev \& Morfill (2004) as $v_{d} \sim(8 \sqrt{2 \pi} / 3) R^{2} n_{n} v_{n} m_{n} / m_{d}$, where the subscript $n$ refers to neutrals. With the parameters in table 1 , taking $E_{0} \sim 1.7 \mathrm{~V} \mathrm{~cm}^{-1}$, we have that $U_{i} \sim v_{i}$ and $U_{e} \sim-0.25 v_{e}$.

In this paper we are interested in the development of the DA instability in the case where the dust is warm, that is, where the dust acoustic speed, $c_{d} \sim \lambda_{D i} \omega_{p d}$, is not much larger than the dust thermal speed $v_{d}$. This implies that $\sqrt{\left(T_{i} / T_{d}\right)\left(Z_{d}^{2} n_{d} / n_{i}\right)}$ is not much larger than unity. For the parameters in table 1 , this requires small $T_{i} / T_{d}$. For example, for $T_{d} \sim\left(T_{e} / 1.5\right) \sim 110 T_{i} \sim 3.3 \mathrm{eV}$ (dust thermal speed $v_{d} \sim 18 \mathrm{~mm} \mathrm{~s}^{-1}$ ), 
(a)

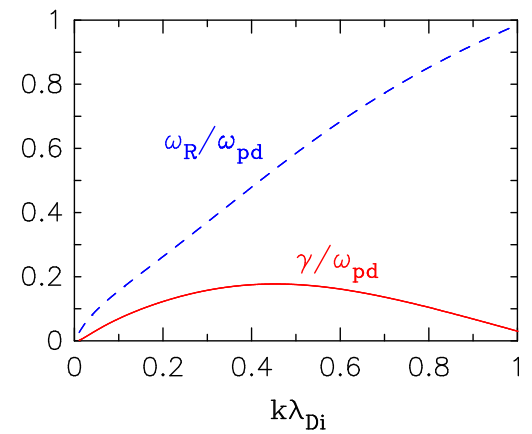

(b)

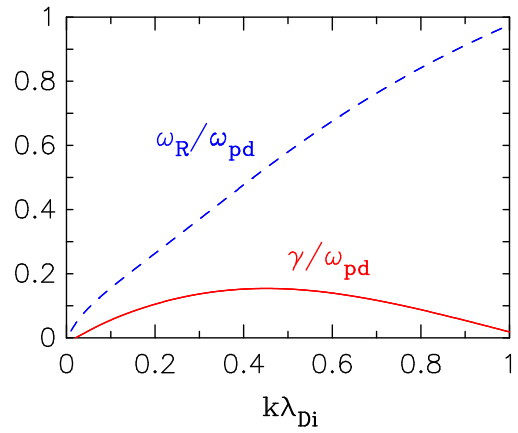

FIgURE 1. Real frequency $\omega_{R}$ (blue, dashed curve) and growth rate $\gamma$ (red, solid curve) normalized to $\omega_{p d}$ versus $k \lambda_{D i}$ obtained by solving (2.2) with $U_{i} / v_{i}=1$ and the dimensionless parameters in $(a)$ table 1 , with $T_{e} / T_{d}=1.5$ and $U_{e} / v_{e}=-0.25$, and $(b)$ table 2, with $T_{d} / T_{i}=1$ and $U_{e}=0$.

$$
\begin{aligned}
& T_{e} / T_{i}=100 \\
& v_{i} / \omega_{p i}=0.5 \\
& v_{e} / \omega_{p i}=30 \\
& Z_{d}=100 \\
& m_{d} / m_{i}=4000 \\
& n_{d} / n_{i}=1 \times 10^{-3} \\
& \omega_{p d} / \omega_{p i}=0.05 \\
& v_{d} / \omega_{p i}=0.01
\end{aligned}
$$

TABLE 2. Reduced parameters.

we have that $c_{d} / v_{d} \sim \sqrt{10}$. (Note that even though $T_{d} / T_{i} \sim 110$, the ratio of the ion thermal speed to the dust thermal speed is large, viz. $v_{i} / v_{d} \sim 2 \times 10^{4} \gg 1$.) The real frequency $\omega_{R}$ and growth rate $\gamma$ obtained by solving (2.2) for the parameters in table 1 , with $T_{e} / T_{d}=1.5, U_{i} / v_{i}=1$ and $U_{e} / v_{e}=-0.25$, are shown in figure $1(a)$.

Because of the very small charge-to-mass ratio of the dust, and correspondingly small ratio of the dust plasma frequency, $\omega_{p d}$, to the ion plasma frequency, $\omega_{p i}$, it is very time consuming to run a numerical simulation of the nonlinear development of this instability using table 1 parameters. However, the essential physics could be reasonably captured by using a set of reduced parameters which reflect the requisite ordering of physical quantities. This includes a dust charge-to-mass ratio which is much smaller than that of the ions, a dust acoustic speed which is much smaller than the ion thermal speed and a growth rate which is much smaller than the ion collision frequency. Thus, we require (1) $Z_{d} m_{i} / m_{d} \ll 1$, (2) $\omega_{p d} / \omega_{p i} \ll 1$, (3) $v_{d}<c_{d} \ll v_{i}$ and (4) wave frequency $\omega \ll v_{i}$. In addition, we need to keep the ratios $v_{i} / \omega_{p i}$ and $v_{d} / \omega_{p d}$ the same as those in table 1. A set of reduced parameters that satisfy these conditions is given in table 2 .

The solution of (2.2) for the real frequency $\omega_{R}$ and growth rate $\gamma$, for the parameters of table 2, with $T_{d}=T_{i}$ and $U_{i}=v_{i}$ is shown in figure $1(b)$. Because $T_{e} \gg T_{i}$ (which is generally representative of low-temperature dusty plasmas in ground-based or microgravity experiments), the electrons are kinetic with $\zeta_{e} \ll 1$, so for simplicity we have taken $U_{e 0}=0$. For the table 2 parameters, $c_{d}=\sqrt{10} v_{d}$, which is approximately the same as in figure $1(a)$. As can be seen, the frequency and linear growth rates 
(a)

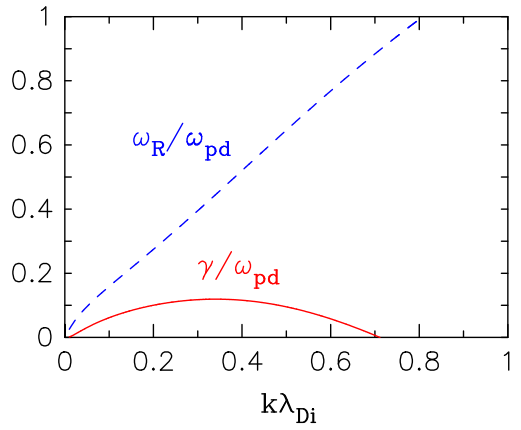

(b)

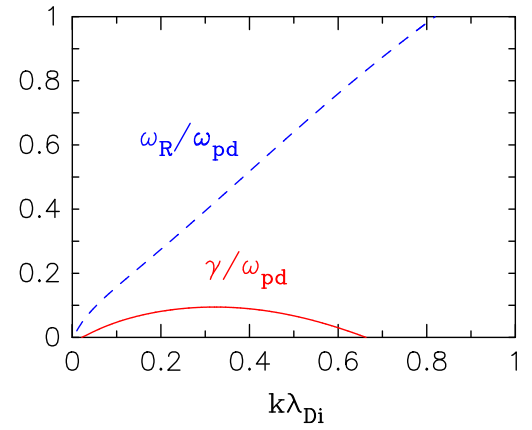

FIGURE 2. Real frequency $\omega_{R}$ (blue, dashed curve) and growth rate $\gamma$ (red, solid curve) normalized to $\omega_{p d}$ versus $k \lambda_{D i}$ obtained by solving (2.2) with the parameters in $(a)$ figure $1(a)$, except that $T_{e} / T_{d}=0.5$, and $(b)$ figure $1(b)$, except that $T_{d} / T_{i}=3$.

look similar in figure $1(a, b)$. This is because the dimensionless parameters that govern the linearly unstable spectrum, which include $U_{i} / v_{i}, c_{d} / v_{d}, v_{i} / \omega_{p i}$ and $v_{d} / \omega_{p d}$, are approximately the same for both cases, and $T_{e} / T_{i} \gg 1$ in both cases. Thus, we will use table 2 parameters for the simulations in the next section.

It is expected from previous simulations of the ion-dust streaming instability that its nonlinear development is associated with an increase in $T_{d}$ (e.g. Winske et al. 1995; Winske \& Rosenberg 1998; Winske 2004; Fu \& Scales 2012). For comparison with the simulation results in the next section, where it will be shown also that $T_{d}$ increases, here we show the results of linear theory for larger $T_{d}$. As $T_{d}$ increases, the maximum growth rate of the linear instability shifts to smaller $k$, i.e. longer wavelength. This may be due to increased dust Landau damping at larger $k$ as the dust thermal speed $v_{d}$ increases (Rosenberg et al. 2008). Figure 2(a) shows the solution of (2.2) for the same parameters as in figure $1(a)$, but with warmer dust, namely $T_{d} \sim 2 T_{e} \sim 10 \mathrm{eV}$, for which the dust acoustic speed $c_{d} \sim 1.8 v_{d}$. As can be seen, maximum growth of the instability has shifted to longer wavelength. Figure 2(b) shows the solution of (2.2) for the reduced parameters used in figure $1(b)$, and for warmer dust with $T_{d} \sim 3 T_{i}$, for which the dust acoustic speed is also $c_{d} \sim 1.8 v_{d}$. As expected, the behaviour of the unstable spectrum for larger $T_{d}$, where $c_{d} \sim 1.8 v_{d}$, appears similar in figure $2(a, b)$ for reasons discussed in the previous paragraph.

\section{Simulations}

The simulations were performed with a 1D electrostatic PIC code with periodic boundary conditions. The plasma species in the PIC simulation consists of singly charged ions that stream in the $x$-direction relative to multiply charged heavy dust grains that are stationary in the simulation reference frame. Because we will be considering the parameter range of figure $1(b)$, where $\zeta_{e} \ll 1$ appears to hold reasonably well for the unstable spectrum, the electrons are treated as Boltzmann distributed and just provide charge neutrality. The ions and dust are treated as particles, and both particle species collide with background neutrals. A Langevin scattering operator, with a constant collision frequency for each charged species, is used to model collisional effects (Gillespie 1993). The numerical implementation of the scattering operator is well established and is described in (Lemons et al. 1995; Winske \& Rosenberg 1998). There is a background electric field $\boldsymbol{E}_{0}=E_{0} \boldsymbol{x}$, whose 


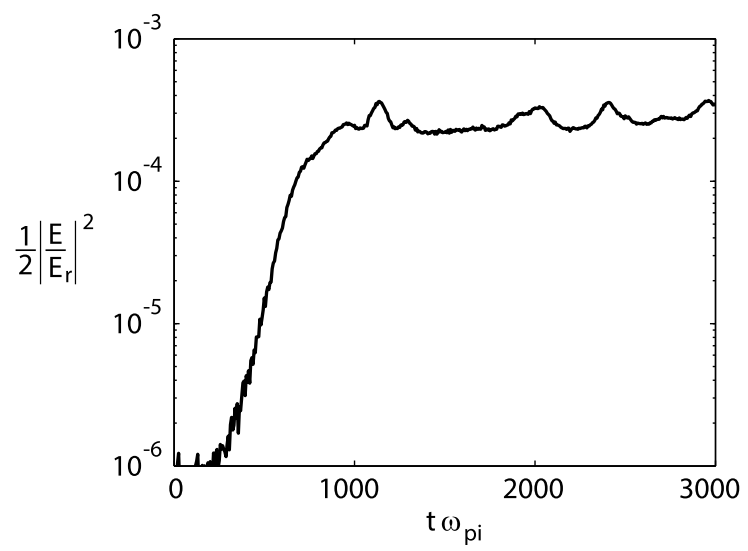

FIGURE 3. Average wave electric field energy density versus time for Case I simulation.

magnitude is set such that the corresponding electric force on the ions balances the ion-neutral collisional force to maintain a particular ion drift velocity $\boldsymbol{U}_{i}=U_{i} \boldsymbol{x}$ during the growth phase of the instability. For this purpose we use the approximate expression $E_{0}=m_{i} v_{i} U_{i} / e$. A nonlinear Poisson equation is used to obtain the self-consistent wave potential $\phi$. Consistent with the Boltzmann approximation, the electron number density $n_{e}$ assumes the form $n_{e}=n_{e 0} \mathrm{e}^{e \phi / T_{e}}$. Together with the ion and dust charge densities, the Poisson equation can then be solved by standard iterative means (Hockney \& Eastwood 1981).

Quantities in the simulation are normalized to ion quantities at the beginning of the simulation. Temperatures and drift speeds are normalized to $T_{i 0}$ and $v_{i 0}$, which are the initial ion temperature and thermal speed, respectively. Length scales are normalized to the ion Debye length $\lambda_{D i}$ evaluated at $T_{i 0}$ and the initial ion density, while time scales are normalized to the ion plasma frequency $\omega_{p i}$ at the beginning of the simulation. The wave electrostatic potential energy $e \phi$ is normalized to $T_{i 0}$ (in energy units) and the wave electric field $\tilde{E}$ is normalized to $E_{r}=T_{i 0} / e \lambda_{D i}$. In this $1 \mathrm{D}$ simulation, the system length is $128 \lambda_{D i}$ and is divided into 256 computational cells. To reduce the numerical noise, the ions and dust are modelled assuming approximately 26 million macroparticles for each species.

Case I. For the first set of simulations, which we call Case I, we use the reduced set of dimensionless parameters given in table 2. The initial drift speed of the ions is taken to be the initial ion thermal speed $v_{i 0}$, while the dust has zero drift. The initial dust temperature and the temperature of the neutrals are both taken to be $T_{i 0}$. Figure 3 shows the average wave electric field energy density as a function of time for this case. The system appears to be in a phase of rapid growth from $t \omega_{p i} \sim 250-600$; this is roughly about three linear growth times, since from figure $1(b), \gamma / \omega_{p d} \sim 0.16$, which implies that $\gamma / \omega_{p i} \sim 0.008$. An estimate of the growth rate in the linear phase of the simulation can be obtained by calculating the logarithmic slope of $\tilde{E}^{2}$ between the times $400 / \omega_{p i}$ and $600 / \omega_{p i}$. This yields $\gamma \sim 0.0065 \omega_{p i}$, in reasonable agreement with the analytic estimate of the maximum growth rate shown in figure $1(b)$.

Figure 4 shows the time histories of the ion and dust temperatures and drift speeds for this case, all averaged over the simulation domain. As can be seen in figure 4(a,b), the ion temperature $T_{i}$ and ion drift speed $U_{i}$ remain approximately constant, while figure $4(c, d)$ show that the dust temperature $T_{d}$ increases to about 2.5-3 times its 

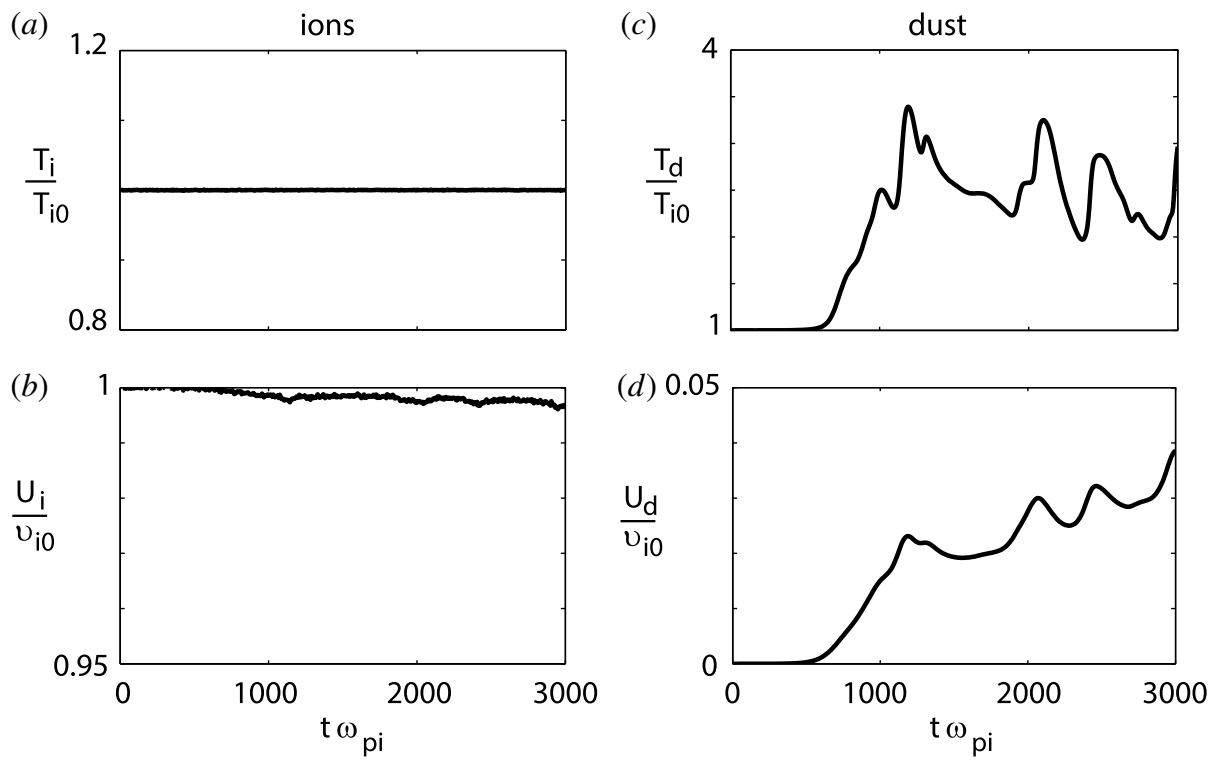

FIGURE 4. Temperature and drift speed versus time for Case I simulation: (a) ion temperature, $(b)$ ion drift speed, $(c)$ dust temperature, $(d)$ dust drift speed.

initial value, and the dust drift speed $U_{d}$ increases to about $\sim 0.02 v_{i 0}$. With a dust temperature of $T_{d} \sim 3 T_{i 0}$, the dust acoustic speed is near the dust thermal speed, with $c_{d} \sim 1.8 v_{d}$.

The panels in figure 5 show ion and dust phase-space plots, along with the spatial structure of the wave electrostatic potential, and the corresponding electric field, at $t \omega_{p i}=400$, which is during the rapid wave growth phase, and at $t \omega_{p i}=1000$, which is in the nonlinear phase. As can be seen from the ion and dust phase-space plots in the nonlinear phase at $t \omega_{p i}=1000$, the dust appears to get trapped by the waves, while the ions do not. The trapping condition is given in (Drummond et al. 1970; Winske 2004) as

$$
Z_{j} e \phi \sim \frac{m_{j}}{2}\left(\frac{\omega_{\max }}{k_{\max }}-U_{j}\right)^{2}
$$

where $U_{j}$ is the mean velocity of the trapped species and $\omega_{\max } / k_{\max }$ is the phase speed of the maximally unstable wave. For the dust, we have assumed that initially $U_{d}=0$ and, taking roughly $\omega_{\max } / k_{\max } \sim c_{d}$, we have that

$$
\frac{e \phi}{T_{i 0}} \sim \frac{1}{2} \frac{Z_{d} n_{d}}{n_{i}} .
$$

For the ions, the initial ion drift $U_{i}=v_{i 0}$ is much larger than the wave phase speed, so that

$$
\frac{e \phi}{T_{i 0}} \sim \frac{1}{2} .
$$

Thus, the condition for dust trapping depends roughly on the magnitude of the relative dust charge density, while the condition for ion trapping is constant for the case of fixed initial ion drift speed $U_{i} / v_{i 0}$ which we consider here. For case I, $Z_{d} n_{d} / n_{i}=0.1$ 
(a)

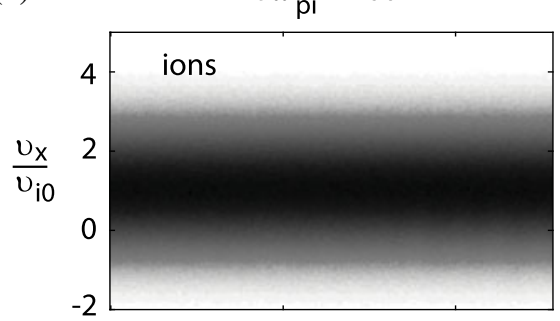

(b)
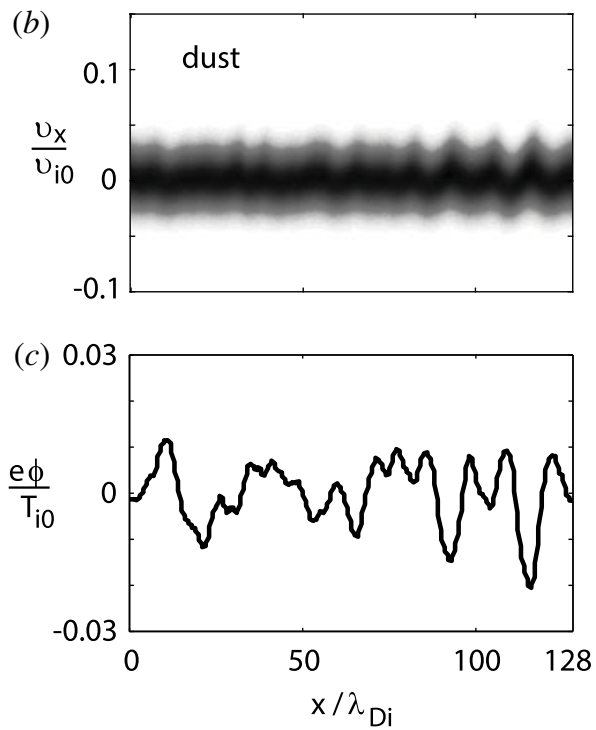

(d)
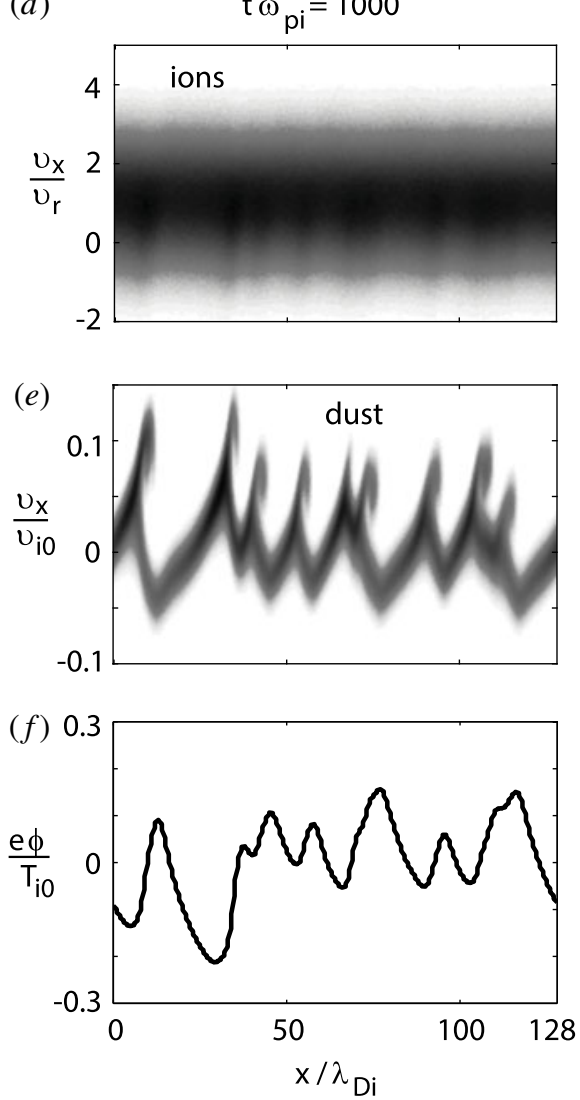

FIGURE 5. Phase-space plots and spatial structure of the wave potential for two different times in the simulation for Case I: $t \omega_{p i}=400(a-c)$ and $t \omega_{p i}=1000(d-f)$. The ion phasespace plots are shown in $(a, d)$, the dust phase-space plots in $(b, e)$ and the wave potential in $(c, f)$.

(see table 2), so the condition (3.2) implies that $e \phi / T_{i 0} \sim 0.05$ in order for dust to get trapped. This can be satisfied with the average values shown in figure $5(f)$. On the other hand, from figure $5(f)$ we see that the wave potential is not large enough to satisfy (3.3) for ion trapping. Thus, we expect dust trapping, but not much ion trapping, which is consistent with phase-space plots shown in figure $5(e, d)$.

The dust phase-space plots in figure $5(b, e)$ suggest that there is a dominant wavelength in the linear growth phase and a different dominant wavelength in the nonlinear phase. In the linear phase, there appears to be roughly $\sim 11$ wavelengths (although irregular) in the simulation length (obtained by counting the peaks in $\phi$ in figure $5 c$ ), implying that $k \lambda_{D i} \sim 2 \pi(11 / 128) \sim 0.5$. In the nonlinear phase, the dust structures shown in figure $5(f)$ suggest a longer dominant wavelength (again somewhat irregular) with roughly seven wavelengths, implying that $k \lambda_{D i} \sim 0.3$. Because the ions do not appear to be trapped but still have a drift due to the external electric field, this suggests that there may be a secondary dust acoustic instability in the nonlinear phase. In this phase, the dust temperature has increased by about a factor of three due to dust trapping and heating by the waves. Thus, we would expect that the wavelength of a secondary DA instability would shift to longer wavelengths. 
This is consistent with the linear theory results shown in figure $2(b)$ for this case with $T_{d} \sim 3 T_{i 0}$, where the maximum growth of the DA instability is roughly around $k \lambda_{D i} \sim 0.3$. In order to pin down in more detail the characteristics of this possible secondary instability, future work will analyse the ion and dust distribution functions and use the results in a linear theory analysis, similar to what has been done recently for the nonlinear phase of the Buneman instability in Jain, Umeda \& Yoon (2011). In the latter paper, it was shown that wave spectra in the nonlinear phase of the Buneman instability could be be related to instantaneous particle distributions using linear theory.

For the parameters we are considering, the ion and dust collision rates are relatively large, and it is possible that the ion and dust distribution functions could become thermalized owing to collisions with neutrals modelled here using the Langevin operator. This might occur if the collision rate is comparable to or larger than the bounce frequency of a particle in the wave potential. The bounce frequency is given by (e.g. Nishikawa \& Wakatani 1990)

$$
v_{b} \sim\left(\frac{Z_{j} e k_{\max } \tilde{E}}{m_{j}}\right)^{1 / 2},
$$

where $\tilde{E}$ is the wave electric field. In the normalized units of our simulations, the ratio of $v_{b}$ to the ion plasma frequency is

$$
\frac{v_{b}}{\omega_{p i}} \sim\left(k_{\max } \lambda_{D i} \frac{Z_{j} m_{i}}{m_{j}} \frac{\tilde{E}}{E_{r}}\right)^{1 / 2} .
$$

Thus, for the dust,

$$
\frac{v_{b}}{\omega_{p d}} \sim\left(k_{\max } \lambda_{D i} \frac{n_{i}}{Z_{d} n_{d}} \frac{\tilde{E}}{E_{r}}\right)^{1 / 2},
$$

which scales inversely with the relative dust charge density. For this case, from figure 3 the average magnitude of the normalized wave electric field $\tilde{E} / E_{r} \sim 0.025$ in the nonlinear phase. For the dust, we then have $v_{b} / \omega_{p d} \sim 0.27$, which is comparable to the dust-neutral collision frequency $v_{d} / \omega_{p d}=0.2$. Thus, while the waves are trapping and heating the dust, collisions are also cooling and tending to thermalize the dust grains. Since in this case we would expect the dust distribution to be near a Maxwellian, it may indeed be the case that a secondary instability in the nonlinear phase could be described by linear theory using a Maxwellian for the dust and a shifted Maxwellian for the ions. However, as mentioned above, the detailed investigation of this topic is left for future work.

Case II. From (3.2), it can be seen that as the relative charge density carried by the dust increases, the magnitude of the wave potential required for dust trapping also increases. And, as the wave potential increases, ion trapping could also occur, in contrast to Case I where the wave potential in the nonlinear phase is too small for ion trapping to occur. To investigate this, we reduce $Z_{d}$ and increase the initial $n_{d} / n_{i}$ in such a way as to increase $Z_{d} n_{d} / n_{i}$ but keep $Z_{d}^{2} n_{d} / n_{i}$ the same as in Case I. We keep the other quantities the same as in Case I, so that other dimensionless quantities including $c_{d} / v_{d}, v_{d} / \omega_{p d}, \omega_{p d} / \omega_{p i}$ and $v_{i} / \omega_{p i}$ are the same as in Case I. Thus, taking 


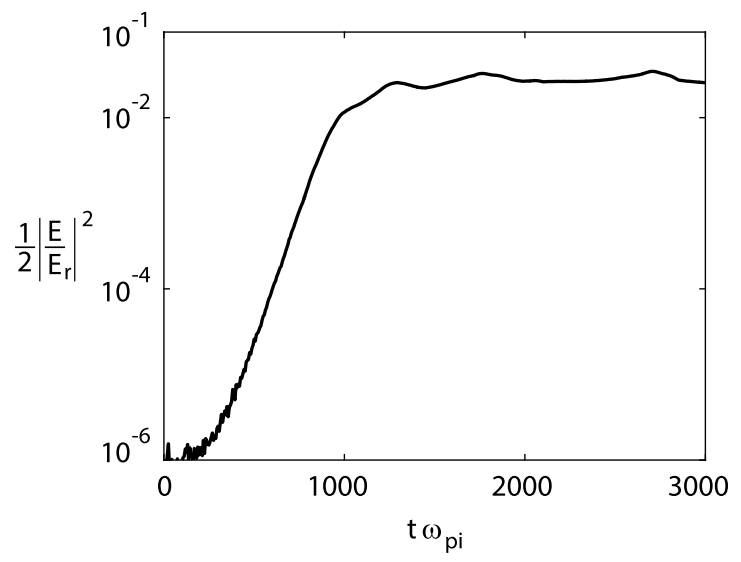

FIGURE 6. Average wave electric field energy density versus time for Case II simulation.

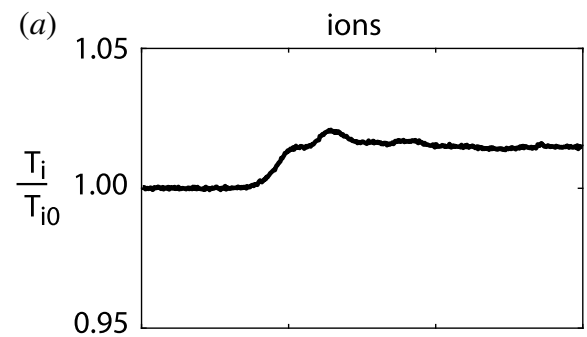

(c)

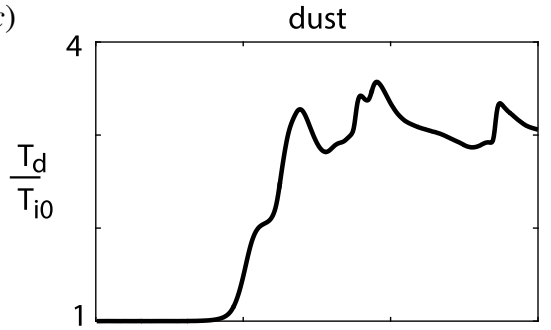

(b)
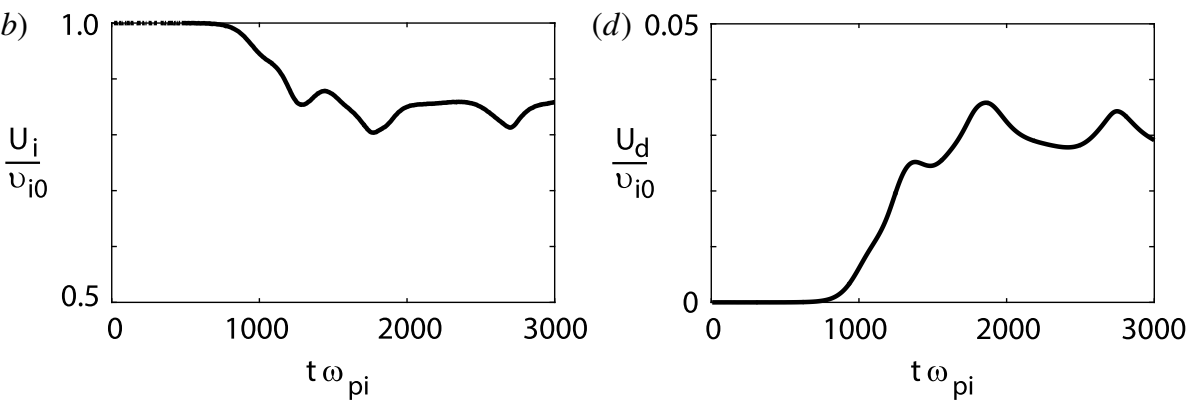

FIgURE 7. Temperature and drift speed versus time for Case II simulation: $(a)$ ion temperature, $(b)$ ion drift speed, $(c)$ dust temperature, $(d)$ dust drift speed.

the same initial ion drift $U_{i}=v_{i 0}$, the linear growth rate for the DA instability would be the same as in Case I.

Thus, we take $Z_{d}=12.5$ and $n_{d} / n_{i}=0.064$, so that $Z_{d} n_{d 0} / n_{i 0}=0.8$, which is eight times as large as in Case I. We point out that this relative dust charge density is close to that in table 1 , where $Z_{d} n_{d} / n_{i}=0.75$. Thus, the condition for dust trapping given by (3.2) would be similar for the parameters of table 1. Figure 6 shows the average wave electric field energy density and figure 7 shows the time histories of the ion and dust temperatures. As can be seen from figure 6, the wave energy density is considerably larger than in Case I, by about two orders of magnitude. Figure 7 shows that the ion temperature increases by a small amount for this case, while the dust temperature 
(a)

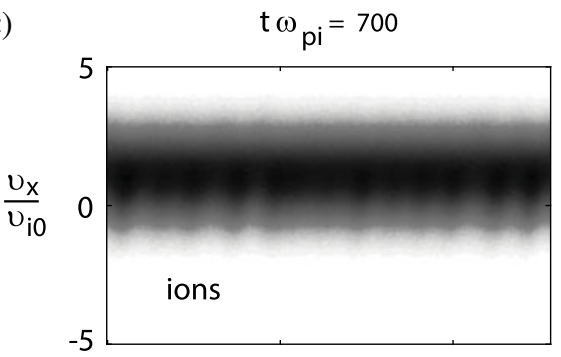

(b)

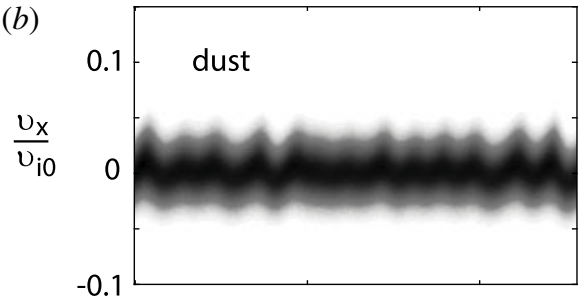

(c)

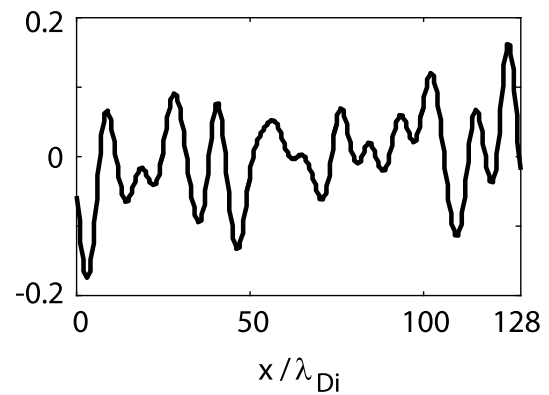

(d)

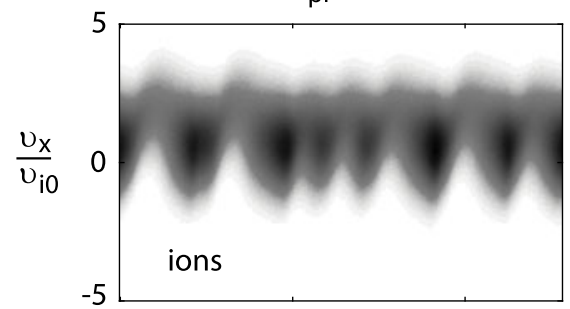

(e)

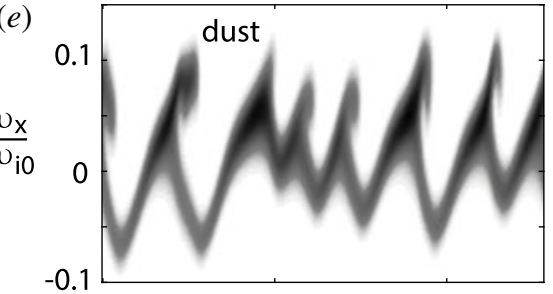

(f)

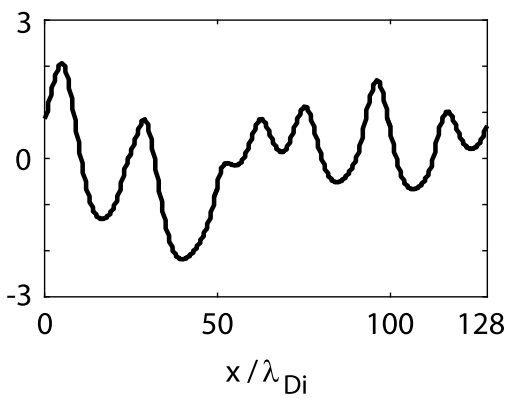

FIGURE 8. Phase-space plots and spatial structure of the wave potential for two different times in the simulation for Case II: $t \omega_{p i}=700(a-c)$ and $t \omega_{p i}=1300(d-f)$. The ion phasespace plots are shown in $(a, d)$, the dust phase-space plots in $(b, e)$ and the wave potential in $(c, f)$.

increases by a factor of about 2.5-3 similarly to Case I. Figure 8 shows the ion and dust phase-space plots and spatial structure of the wave potential at times $t \omega_{p i}=700$ in the linear phase and $t \omega_{p i}=1300$ in the nonlinear phase. From figure $8(f)$, it can be seen that $e \phi / T_{i 0}$ is large enough to satisfy (3.3), so that some ion trapping could occur. In addition, the condition (3.2) for dust trapping, $e \phi / T_{i 0} \sim 0.4$, is also satisfied. Thus, in this case, it appears that both dust and ion trapping could occur. This is consistent with the increases in ion and dust temperatures shown in figure $7(a, c)$, and with the phase-space plots in the nonlinear phase shown in figure $8(d, e)$. In addition, the mean speed of the ions is pulled to lower speeds, as shown in figure $7(b)$. Note that because the DA waves have to reach a higher amplitude than in Case I for dust trapping to occur, the nonlinear stage starts later than Case I, at about $t \omega_{p i} \sim 1000$.

We can estimate the bounce frequencies from (3.4), using an average normalized wave electric field magnitude of about 0.2 from figure 6. For the ions, this yields a bounce frequency of about $v_{b} \sim 0.3 \omega_{p i}$, while, for the dust, $v_{b} \sim 0.017 \omega_{p i} \sim 0.3 \omega_{p d}$. Both of these bounce frequencies are comparable to their respective collision frequencies, so this implies that collisions can play an important role in detrapping the ions and dust particles over a few bounce periods (see also Winske 2004). For the 
ions, there is a relatively small increase in temperature shown in figure $7(a)$. There is also a small decrease of the mean ion drift shown in figure 7(b), while the phase-space plot in figure $8(d)$ shows a small downward shift of the ion speed in spatial regions where the wave potential has minima.

We note that for this case, the linear growth rate, the condition for trapping and the scaling of the normalized bounce frequencies with $Z_{d} n_{d} / n_{i}$ would be similar for the parameters in table 1 with initial $U_{i}=v_{i 0}$ and $c_{d} / v_{d}=\sqrt{10}$. This suggests that the nonlinear development shown for the set of reduced parameters in Case II may give a reasonable model for the nonlinear development of the DA instability for the experimentally representative parameters in table 1 .

\section{Summary}

We have presented 1D PIC simulations of the nonlinear development of the dust acoustic instability in a collisional plasma where the dust is warm, that is, where the dust acoustic speed is not much larger than the dust thermal speed. Both ion-neutral and dust-neutral collisional effects were included in the simulations, along with a background electric field that generates an ion flow that excites the instability. We considered the parameter regime where the ion flow speed is thermal, which may be relevant to dusty plasma experiments in microgravity where electric fields that drive the ion flow can be weaker than in ground-based experiments.

We considered how the nonlinear development of the instability varies as the relative charge density of the dust varies, while other relevant dimensionless parameters remain the same. For the parameters we considered, it was found that when the relative dust charge density is small, the dust can get trapped and heated by the waves, but the ions do not appear to be affected much. In the nonlinear phase, there appears to be a dominant wavelength of the potential structure that is longer than the dominant wavelength in the linear phase. We suggested that this may be due to a secondary dust acoustic instability, which would have longer wavelength than the primary instability due to the elevated dust temperature. The dominant wavelength in the nonlinear phase appears to be consistent with the results of linear kinetic theory, using the initial ion and dust velocity distributions but with larger dust temperature. However, further work will analyse the actual ion and dust velocity distributions in the nonlinear phase and use these in a linear stability analysis of a possible secondary instability. When the relative dust charge density is large, the ions also begin to get trapped in the waves. In this case, while the dust gets heated, it appears that the ions get heated only slightly, along with a small decrease in the ion drift speed.

\section{Acknowledgements}

This work was partially supported by NSF and NASA.

\section{REFERENCES}

Drummond, W. E., Malmberg, J. H., O’Neil, T. M. \& Thompson, J. R. 1970 Nonlinear development of beam-plasma instability. Phys. Fluids 13, 2422-2425.

Fortov, V., Morfill, G., Petrov, O., Thoma, M. et al. 2005 The project 'Plasmakristall-4 (PK-4)' - a new stage in investigations of dusty plasmas under microgravity conditions: first results and future plans. Plasma Phys. Control. Fusion 47, B537-B549.

Fried, B. D. \& Conte, S. D. 1961 The Plasma Dispersion Function. Academic. 
FU, H. \& SCALES, W. 2012 Nonlinear evolution of the dust acoustic instability in artificially created dusty space plasmas. IEEE Trans. Plasma Sci. 40, 1223-1228.

Gillespie, D. T. 1993 Fluctuation and dissipation in Brownian motion. Am. J. Phys. 61, 1077-1083.

Hockney, R. W. \& Eastwood, J. W. 1981 Computer Simulation using Particles. McGraw-Hill.

JAIN, N., UmedA, T. \& Yoon, P. H. 2011 Modeling nonlinear development of Buneman instability with linear dispersion theory. Plasma Phys. Control. Fusion 53, 025010.

Joyce, G., LAmpe, M. \& GAnguli, G. 2002 Instability-triggered phase transition to a dusty-plasma condensate. Phys. Rev. Lett. 88, 095006.

Khrapak, S. A., Ivlev, A. V. \& Morfill, G. E. 2004 Momentum transfer in complex plasmas. Phys. Rev. E 70, 056405.

Khrapak, S. A., Ratynskaia, S. V., Zobnin, A. V., Usachev, A. D. et al. 2005 Particle charge in the bulk of gas discharges. Phys. Rev. E 72, 016406.

Lemons, D. S., Lackman, J., Jones, M. E. \& Winske, D. 1995 Noise-induced instability in self-consistent Monte-Carlo calculations. Phys. Rev. E 52, 6855-6861.

Melzer, A., Homann, A. \& Piel, A. 1996 Experimental investigation of the melting transition of the plasma crystal. Phys. Rev. E 53, 2757-2766.

Merlino, R. L. 201425 years of dust acoustic waves. J. Plasma Phys. 80, 773-786.

MiYamoto, K. 1989 Plasma Physics for Nuclear Fusion. MIT Press.

Nishikawa, K. \& WaKatani, M. 1990 Plasma Physics: Basic Theory with Fusion Applications. Springer.

Rao, N. N., Shukla, P. K. \& YU, M. Y. 1990 Dust-acoustic waves in dusty plasmas. Planet. Space Sci. 38, 543-546.

Rosenberg, M. 1993 Ion- and dust-acoustic instabilities in dusty plasmas. Planet. Space Sci. 41, 229-233.

Rosenberg, M. 1996 Ion-dust streaming instability in processing plasmas. J. Vac. Sci. Technol. A 14, 631-633.

Rosenberg, M., Thomas, E. JR. \& Merlino, R. L. 2008 A note on dust wave excitation in a plasma with warm dust: comparison with experiment. Phys. Plasmas 15, 073701.

Schweigert, V. A., Schweigert, I. V., Melzer, A., Homann, A. \& Piel, A. 1996 Alignment and instability of dust crystals in plasmas. Phys. Rev. E 54, 4155-4166.

Williams, J. D. \& Thomas, E. 2006 Initial measurement of the kinetic dust temperature of a weakly coupled dusty plasma. Phys. Plasmas 13, 063509.

Williams, J. D. \& Thomas, E. JR. 2007 Measurement of the kinetic dust temperature of a weakly coupled dusty plasma. Phys. Plasmas 14, 063702.

WINSKE, D. 2004 Wave drag due to dust acoustic waves in collisional dusty plasmas. IEEE Trans. Plasma Sci. 32, 663-674.

Winske, D., Gary, S. P., Jones, M. E., Rosenberg, M., Chow, V. W. \& Mendis, D. A. 1995 Ion heating in a dusty plasma due to the dust ion-acoustic instability. Geophys. Res. Lett. 22, 2069-2072.

Winske, D. \& Rosenberg, M. 1998 Nonlinear development of the dust acoustic instability in a collisional dusty plasma. IEEE Trans. Plasma Sci. 26, 92-99. 